$\mathrm{XX}$.

\title{
Arbeiten aus dem pharmakologischen Institut der Universität Dorpat.
}

I.

Ueber die physiologischen Wirkungen des

Pseudaconitin (Nepalin)

nach Untersuchungen des Dr. med. C. Ewers

mitgetheilt durch

Prof. Dr. R. Boehm.

Eine genauere Erforschung der physiologischen Wirkungen des Bikhknollen-Alkaloides erschien aus mehreren Grïnden wiinschenswerth. Abgesehen von dem an und für sich schon ausreichenden allgemein wissenschaftlichen Interesse musste eine solche Untersnehung anch praktisch verwerthbare Frichte tragen. Denn bekanntlich wird die aus den Wurzeln von Aconitum ferox erhaltene Base namentlich in England mannigfach als Arzneimittel gebraucht. Zudem gestatten uns die Kenntnisse, die wir nunmehr über die Wirkungen des wirksamen Alkaloides aus Aconitum napellus besitzen, eine präcise Vergleichung beider Alkaloide in physiologischer Hinsicht, die um so mehr erwünscht ist, seitdem man erfahren hat, wie wenig sie sich chemisch von einander unterscheiden. Die chemische Seite der Frage wurde vor der Hand ganz unberiicksichtigt gelassen, weshalb ich auch hier Umgang nelime, darauf näher einzugehen.

Herr Prof. Dragendorff hatte die Güte, aus einer grösseren Menge schöner Bikhknollen das Alkaloid in der nöthigen Menge für unsere Untersuchungen darzustellen. Es stehen von seiner Seite detaillirtere Angaben über die chemischen Eigenschaften dieser Base in Aussicht.

Archiv f. experiment. Pathologie und Pharmakologie. I. Bd. 
Herr Dr. med. C. Ewers, in Gemeinschaft mit welchem ich die physiologische Untersuchung des Pseudaconitins*) im verflossenen Winter angestellt habe, hat die Resultate derselben bereits in seiner Dissertation**) publicirt, auf welche ich hier namentlich mit Beziehmog anf die detaillirten Versuchsprotocolle und die einleitenden historischen Notizen verweise.

Ich werde mich hier auf eine gedrängte Darlegung unserer Ergebnisse beschränken, wobei ich die Wirkungen des dentschen Aconitins als bekannt voraussetzen darf.

Im allgemeinen Wirkungscharakter stimmt das Pseudaconitin mit dem deutschen Aconitin iberein und es wird sich wenig gegen die Behauptung einwenden lassen, dass die vorhandenen Differenzen wesentlich quantitative sind und die Intensität der Wirkung betreffen. Die einzige offenbar qualitative Differenz, die unsere Untersuchungen constatirten, bezieht sich auf die locale Wirkung des Pseudaconitins auf die Schleimhäute und die äussere Haut. Wir haben in dieser Richtung am Pseudaconitin Wirkungen wahrgenommen, die mehreren Sorten deutschen Aconitins vollständig mangelten. Freilich haben Schroff** $)$ u. A. anch diese locale Wirkung auf die sensibeln Nervenenden dem deutschen Aconitin zugeschrieben, und wir können demnach nicht ganz sicher entscheiden, ob nicht auch in diesem Punkte Pseudaconitin und Aconitin analoge Wirkungen äussern können.

Einige interessante Seiten der Aconitinwirkung, namentlich die Wirkung anf die Respiration, die auch beim deutschen Aconitin noch nicht näher verfolgt sind, haben wir bei dieser Gelegenheit eingehender untersucht. Insofern bilden diese Blätter anch eine Ergầnzung der Kenntniss der Aconitinwirkung überhaupt.

Im äusseren Wirkungsbild des Pseudaconitins bei Versuchen an Katzen und Kaninchen springt nichts so sehr in die Augen als die in wenigen Minuten nach der Vergiftung sich einstellenden Athmungsbeschwerden der Thiere, wenn man das Gift in hinreichender Menge in eine Vene oder in das subcutane Zellgewebe injicirt hat. Bei diesem Vergiftungsmodus erliegen die Versuchsobjecte in 2 bis 40 Minuten dem Erstickungstode und die hierzu erforderliche Giftdose beträgt nur 0,5 Milligramm $(=1 / 120$ Gran). Von deutschem Aconitin sind zur Erzielung dieser Wirkung wenigstens 10 Milligramm,

*) Wir bedienen uns in der Folge ausschliesslich der Bezeichnung „Pseu daconitin" für das aus Aconitum ferox dargestellte Alkaloid.

**) Ueber die physiologischen Wirkungen des aus Aconitum ferox dargestellten Aconitin. Dorpat 1873.

***) Prager Vierteljahrsschrift. XLII. 1854. 
also das 20 fache Quantum erforderlich. Neben der Dyspnöe sind Salivation und Muskelschwäche bis zur Paralyse nie fehlende Intoxicationserscheinungen. Bei der Application des Giftes per os (natürlich bedarf es hierzu grosser Mengen) oder oesophagum, treten zunächst die localen Wirkungen des Giftes auf die Sehleimhäute in den Vordergrund; sie bestehen in Schmerzensäusserungen, Lecken und Kauen und Erbrechen. Wird letzteres durch Oesophagusligatur verhindert, so erfolgt der Tod unter den gleichen dyspnoischen Erscheinungen wie bei Injection ins Blut oder in die Gewebe, wenn auch in viel längerer Zeit $(1-8$ Stunden). Niemals sahen wir gastroenteritische Erscheinungen im Gefolge der Pseudaconitinvergiftung weder miter den Symptomen während des Lebens, noch bei den Leichenöffnungen.

Bei einer genaueren Schilderung der Wirkung des Pseudaconitins auf Frösche motisste man alles das beinahe wörtlich wiederholen, was ich*) und Wartmann über die Wirkung des deutschen Aconitins mitgetheilt haben. In den seitlichen Bauchmuskeln beginnende und von da auf alle anderen willkürlichen Muskeln sich ausbreitende fibrilläre Zuckungen, allgemeine Paralyse und Erlöschen der Reflexe sind hierbei die constanten und wesentlichen Erscheinungen.

Doch ist auch hier die höhere Wirksamkeit des Pseudaconitins unverkennbar. Haben wir für das deutsche Aconitin 0,5 Milligramm als die Minimaldose festgestellt, nach welcher die Lähmung des Thieres im Zeitraum einer Stunde eriolgt, so beträgt diese für das Pseudaconitin 0,03 Milligramm. Das Verbältniss der Wirkungsintensität beider Gifte in Zahlen ausgedrickt ist demnach für diesen Punkt $17: 1$; also annähernd das nämliche wie bei Säugethieren.

Die Erregbarkeit der motorischen Nerven und der Muskelsubstanz fanden wir durch das deutsche Aconitin im Gegensatz zu Asch arumo w*) $^{*}$ and $\mathrm{Weyland}{ }^{* *}$ ) nicht verändert. Auch beim Pseudaconitin sind Nerven und Muskeln noch lange für schwache Reize empfindlich, nachdem schon allgemeine Paralyse eingetreten ist. Doch tritt hier bald ein Zeitpunkt ein, wo es nicht mehr gelingt, durch Nervenreizung Muskelzuckungen auszulösen. Vergiftet man mit grösseren Dosen (ca. 2,5 Milligramm z. B.), so findet man die Erregbarkeit der Nerven nach 28-30 Minuten erloschen. Kleinere

*) Untersuchungen über die physiolog. Wirkungen des deutschen Aconitins. Verhandlg. der phys. med. Gesellsch. zu Würzburg. 1872.

**) Arch. f. Anat. u. Physiologie. 1866.

***) Eckhard's Beiträge zur Anatomie und Physiologie. Bd. V. 
Giftmengen (0,05-1,0 Milligramm) führen das gleiche Resultat in 40 Minuten bis 5 Stunden herbei. Gerade wegen des Gegensatzes, der sich hierin im Vergleich mit der Wirkung des deutschen Aconitins ausspricht, wurde diesem Punkte eine besondere Aufmerksamkeit zugewandt. Es liess sich zunächst leicht constatiren, dass der VerIust der Erregbarkeit in der That eine Wirkung des Pseudaconitins und keine zufällige Erscheinung war. Denn der Nervus ischiadicus eines Frosches, dessen entsprechende Arteria femoralis vor der Vergiftung unterbunden wird, behält seine Irritabilität so lange wie ein normaler durchschnittener Nerv. Es fragte sich daher nur, ob man an diesem Verhalten des mit Pseudaconitin vergifteten Nervmuskelpräparates wirklich etwas von dem Wesen der Wirkung des deutschen Aconitin Abweichendes erblicken darf. Meine Versuche mit deutschem Aconitin hatte ich an gesunden Exemplaren von Rana esculenta angestellt. Hier in Dorpat ist diese Art nicht zu haben. Wir mussten uns daher mit allerdings recht kräftigen Repräsentanten der Rana temporaria begnügen. Dieser Umstand aber legte es nahe, das Verhalten dieser Thiere auch gegen deutsches Aconitin zu prüfen, und diese Vorsichtsmassregel rechtfertigte sich. Denn es stellte sich alsbald heraus, dass das deutsche Aconitin und zwar das gleiche Präparat, das ich zu meinen Untersuchungen mit Wartmann in Würzburg benutzte - bei den Dorpater Fröschen ebenso die Nervenirritabilität aufhebt wie das Psendaconitin. Was folgt nun aus diesen widerspruchsvollen Resultaten? Vor Allem, so glaube ich, eine neue Mahnung, mit der Verallgemeinerung von Versuchsergebnissen vorsichtig zu sein. Unsere Versuche mit dem deutschen Aconitin hatten wir hauptsächlich wegen unserer von Ascharumow*) und Weyland**) abweichenden Resultate in soleher Anzahl und unter so mannigfach variirten Bedingungen angestellt, dass wir das damalige Ergebniss auch heute noch aufrecht halten müssen. Ebenso sicher haben wir uns aber ron dem entgegengesetzten Verhalten der hiesigen Frösche überzeugt. Es bleibt also nichts Anderes iibrig, als anzunehmen, dass die beiden angewandten Froscharten sich in dieser Beziehung verschieden verhalten, dass aber deutsches Aconitin und Pseudaconitin anch in dieser Richtung keine Wirkungsdifferenzen zeigen. Wahrscheinlich, wird es sich auch hier, wie beim Veratrin und Carare, um eine Lähmung der intramusculären Nervenendapparate handeln; denn die Muskelsubstanz selbst bleibt erregbar und eine Veränderung der Nerven-

*) 1. c. **) 1. c. 
faser selbst ist nach allen vorliegenden Erfahrungen kaum mehr anzunehmen. Für die sofort nach der Vergiftung eintretende Lähmung der willkürlichen Bewegungen kommt diese Veränderung der Nervenirritabilität ảber nicht in Betracht. Die Thiere sind schon vollständig gelähmt zu einer Zeit, wo noch schwache elektrische Nervenreizung prompte Muskelzuckungen auslöst. Die Paralyse bei der Aconitin- und Pseudaconitinvergiftung muss daher als eine spinale aufgefasst werden.

Eine Veränderung der Muskelzuckungscurve konnten wir ebenso wenig bei der Vergiftung mit Pseudaconitin ermitteln, als dies mir mit Wartmann beim deutschen Aconitin gelungen war.

Ueber die Wirkungen des Pseudaconitins auf das Froschherz haben wir dem nichts hinzuzufügen, was bereits rom deutschen Aconitin in dieser Beziehong bekannt ist, nur kommt auch hier die quantitive Differenz beider Gifte in dem oben angegebenen Verhältniss von ca. $17: 1$ zur Geltung.

Die Respirationsstörungen an Säugethieren sind unstreitig die interessanteste Erscheinung der Aconitin- und Pseudaconitinvergiftung. Sie verdienten vor Allem ein eingehenderes experimentelles Studium. In unserer mehrfach erwähnten Arbeit über das deutsche Aconitin kamen wir zu dem Schluss, dass es sich um eine centrale Affection der Respirationsmechanismen handle. Näher waren wir auf diese Frage damals nicht eingegangen. Diese Liicke sollte nun ausgefillt werden. Es hat sich herausgestellt, dass qualitativ auch in dieser Sphäre Aconitin und Pseudaconitin identisch wirken und das Folgende gilt deshalb auch für beide Gifte in gleicher Weise.

Das einzig passende Versuchsobject für derartige Versuche sind bekanntlich Kaninchen. Der Mangel an solchen Thieren hat es verschuldet, dass wir die interessanten Resultate dieser Untersuchung in einigen Punkten nicht weiter verfolgen konnten.

Ueber unsere Methode ist wenig zu sagen. Wir brachten die Trachealcanïle mit einem Gabelrohr in Verbindung, dessen eine Zinke mit einem Marey'schen Cardiographen communicirte, der die Frequenz der Athemzitge an der Kymographiontrommel registrirte. In anderen Versuchen wurde das Thier einfach auf den Tisch gesetzt und die Athemzüge an den Bewegungen der Lippen beobachtet und gezählt.

Es muss noch vorausgeschickt werden, dass eine genantere Beobachtung der Athmungsphänomene nur dann möglich ist, wenn man das Gift subcutan injicirt. Spritzt man es in die Vene ein, so 
erfolgt der Tod so rasch, dass die dyspnoëtischen Symptome kaum zum deutlichen Ausdruck gelangen können.

Ein durch subcutane Injection von 0,5-2,0 Milligramm Pseudaconitin vergiftetes Kaninchen zeigt nach ea. 5 Minuten die ersten unverkennbaren Intoxicationserscheinungen. Sie bestehen in der Regel in Zittern, Salivation und Kaubewegungen. Zählt man die Athemzïge, so beobachtet man nach 10 Minuten eine deutliche Verlangsamung derselben, die stetig zunimmt, bis das Thier in ca. 90 Minuten dem Gift erliegt. Die Respirationsfrequenz nicht tracheotomirter Kaninchen ist sehr hoch und beträgt 90-140 sehr oberflächliche Athemzüge in der Minute. Durch die Vergiftung mit Aconitin oder Pseudaconitin reducirt man diese Zahl auf 5-20 Respirationen in der Minute, die den Charakter äusserster Dyspnöe an sich tragen. Sie sind dwrch lange und absolut apnoische Pausen von einander getrennt. Die Erscheinungen an tracheotomirten Thieren sind wesentlich die gleichen, nur ist die anfängliche Frequenz der Respiration in der Regel etwas geringer. Der Tod, der fast nie später als 30 Minuten nach der Vergiftung erfolgt, ist ein exquisiter Erstickungstod.

Wir haben in unserer Abhandlung über das deutsche Aconitin die Bemerkung gemacht, das Vagusdurchschneidung am Halse das Zustandekommen der Suffocationsercheinungen nicht aufhalte. Diese Behauptung muss nach unseren nunmehrigen Erfahrungen modificirt werden.

Durchschneidet man nämlich die NN. vagi eines Kaninchens nach der Vergiftung mit Aconitin oder Pseudaconitin in dem Zeitpunkte, wo die Dyspnöe eben anfängt ihren Höhepunkt zu erreichen, so wird man durch die höchst auffallende Thatsache überrascht, dass nun die Athmang plötzlich ihren dyspnoischen Charakter verliert, regelmässig; und zugleich wieder bedeutend frequenter: wird. Wir lassen ein Beispiel folgen. Die Respiration eines Kaninchens vor der Vergiftung betrug 90. 18 Minuten nach der Injection von 0,5 Milligramm Psendaconitin ist sie auf 16 gesunken. In der 18. Minute werden beide Nervi vagi durchtrent und in der 19. hat sich die Respiration wieder auf 79 in der Minute erhoben und alle Dyspnöe ist verschwunden. Nachdem sich uns dieses Resultat in vielen Versuchen constant dargeboten hatte, konnten wir an keine Täuschung mehr denken. Die Vagusdurchschneidung hob also die Dyspnöe auf, während sie doch sonst beim normalen Thiere gerade Dyspnöe erzengt. 
Eine entsprechende Variirung der Versuchsanordnungen liess uns zu obigem noch folgende Resultate hinzuftigen.

1) Die Vagusdurchschneidung verzögert wohl den Erstickungstod, hebt ihn aber nicht auf. Erfolgte er ohne sie nach 30 Minuten, so erliegt ihm das Thier mit durchschnittenen Vagis nach 2 bis 3 Stunden.

2) Die Vagusdurehschneidung hat keinen Effect auf die Aconitinoder Pseudaconitin-Dyspnöe, wenn sie kurz vor dem Tode des Thieres, also in den letzten 5 Minuten ausgeführt wird. Sie muss im Anfange, wenn die Dyspnöe gerade beginnt sehr heftig zu werden, vorgenommen werden.

3) Es genügt häufig zur Beseitigung der Dyspöne, wenn man nur einen Vagus durchschneidet.

4) Durchsehneidet man die NN. vagi vor der Vergiftung mit Aconitin oder Pseudaconitin, so wird die Giftwirkung verzögert. Die Erstickung tritt viel langsamer ein als ohne durchschnittene NN. vagi.

5) Die NN. laryngei superiores und inferiores sind bei der Wirkung des Aconitins und Pseudaconitins ganz unbetheiligt. Ihre Durchschneidung vor und nach der Vergiftung ändert nichts an der Erscheinungsreihe.

6) Den gleichen Dienst wie Vagusdurehtrennung thut bei der Aconitin- und Pseudaconitinvergiftung das Atropin.

Diese Facta sind nur dann einigermassen. verständlich, wenn man dem Nerrus ragus einen antagonistischen Einfluss auf die Respirationsbewegungen einräumt, wenn man, mit anderen Worten, ihn sowohl als Hemmungs- wie als Beschleunigungsnerven der Athmung gelten lässt. Eine solche Auffassung ist aber heute zu Tage nicht mehr ganz ohne thatsächlichen Boden. Burk har d $t^{\prime *}$ ) und Bre u er's**) Arbeiten sprechen in gleicher Weise dafür und gegen die Annahme Rosenthal's, dass im Vagus nur inspirationsbeschlemigende und exspirationshemmende Fasern verlaufen.

Wenn man nun annimmt, dass bei schwacher Reizung die besehleunigendeu Fasern im Sinne Rosenthal's, bei starker die hemmenden das Uebergewicht haben, und dass das Aconitin und Pseud-1 aconitin die sensibeln Enden der Vagi in der Lunge stark reizen, so wird die Wirkung der Vagusdurchschneidung bei der Aconitinresp. Pseudaconitinvergiftung befiiedigend erklärt, ohne dass man sich allzuweit auf hypothetisehes Gebiet begiebt. Wir können diese

*) Pflüger's Archiv. I.

**) Wiener Sitzgsber. Naturw. Kl. Bd. VIII. 
Auffassung noch durch eine weitere Beobachtung stïtzen. In mehreren Fällen sahen wir bei Kaninchen, deren Dyspnöe durch Vagusdurchtrennung gehoben war, dyspnoëtische Anfälle zurickkehren, sobald man den centralen Vagusstumpf auf die Elektroden brachte und reizte.

Doch damit ist erst die Hälfte der Erscheinungen erklärt. Die andere Hälfte, die nach der Vagusdurchschneidung allmälig wieder eintretende Athemnoth und die antagonistische Wirkung des Atropins, machen es unumgänglich, dass wir auch unsere frühere Annahme von dem Einfluss der Aconitine auf das Athmungscentrum aufrecht erhalten. In welcher Weise aber dieses uns noch so wenig näher bekannte Centralorgan afficirt wird, dariiber enthalten wir uns jeder Discussion. Soviel ist sicher, dass es zuletzt seine Thätigkeit einstellt, - gelähmt wird, und dass das Atropin im Stande ist, diese Wirkung bis zu einem gewissen Grade zu paralysiren. Daraus ergiebt sich auch, dass dieser Stoff das rationelle Gegengift bei der Aconitinvergiftung ist. Dass Atropin auf die Athmungscentren einwirkt, hat schon v. Bezold*) dargethan. Schmiedeberg**) fand, dass es auch die Athmungsbeschwerden zu heben im Stande ist, die mit der Muscarinvergiftung verbunden sind. Indessen sind alle diese Thatsachen physiologisch noch nicht zu erklären. Durch eine grössere Reibe vergleichender Untersuchungen ïber Respirationsgifte könnte vielleicht diese höchst interessante Frage ihrer Lösung näher gerückt werdeu.

$\mathrm{Zu}$ den constanten Symptomen der Aconitin- und Pseudaconitinvergiftung gehört hochgradige Salivation. Es war unsere Absicht, anch den Zusammenhang dieses Phänomens mit den iubrigen Wirkungen des Giftes möglichst vollständig zu eruiren. Es war uns aufgefallen, dass bei mehreren Kaninchenversuchen die Salivation nicht eintrat, wenn zuvor die NN. vagi durchschnitten worden waren, und dass die bereits vorhandene Salivation gleichzeitig mit der Dyspnöe nach der Vagusdurehschneidnng wieder verschwand. Diese Thatsachen machten es wahrscheinlich, dass Dyspnöe and Salivation in ihrem Ursprung etwas Gemeinsames haben, und es lag die Vermuthung nahe, dass etwas Analoges vorliege, wie es 0 ehl $1 * * *$ ) beschrieben hat, der nach centraler Vagusreizung reflectorisch eine vermehrte Salivation eintreten sah. Da Speichelversuche an Kaninchen wegen der Kleinheit der Organe schwer auszuführen sind, so experimentirten wir zur Entseheidung dieser Frage an Hunden

*) Arbeiten aus dem physiol. Instit. zu Würzburg. I. Heft.

**) Das Muscarin etc. Leipzig 1869.

***) Compt. rend. 1864 . S. 336 . 
und Katzen, bei denen wir Canïlen ohne Schwierigkeiten ịn den Speichelgang der Submaxillardrisse einbinden konnten. Doch gelangten wir zu keinem sicheren Resultate. Es trat bei den genannten Thieren eine viel geringere Salivation nach der Pseudaconitinvergiftung ein als bei Kaninchen - wahrscheinlich wohl deshalb, weil schon vor der Vergiftung und Präparation (es wurde wegen seines bekannten Einflusses auf die Speichelsecretion absichtlich kein Curare angewandt) das Thier durch Schreien und Befreiungsversuche, theils auch durch Knebelung u. dgl. auf reflectorischem Wege zuviel Speichel verloren hatte. Nichts destoweniger bleiben die an Kaninchen gemachten Beobachtungen bemerkenswerth und werden vielleicht demnächst den Ausgangspunkt weiterer Versuche bilden.

Der Einfluss des Pseudaconitins auf den Kreislauf der Säugethiere wurde gleichfalls einer genauen Prüfung unterworfen. In diesem Punkte wichen Pseudaconitin und deutșcheś Aconitin einigermassen; ron einander ab, wiewohl man vielleicht bei näherer Ueberlegung auch diese Differenzen auf quantitative Verschiedenheiten wird zurückführen können. Es ist eine geradezu charakteristische Erscheinung der Vergiftung mit dentschem Aconitin, dass wenige Minuten nach der Einspritumg des Giftes in die Vene bei allen Thieren (Kaninchen, Hunden, Katzen) eine enorme Verlangsamung: der Pulsfrequenz eintritt, die in der Regel längere Zeit andauert und Anfangs mit gesteigertem oder wenigstens nicht gemindertem Blutdruck vorhanden ist. Wir sehen dies Phänomen sowohl nach vorangegangener Vagusdurchschneidung, als nach Atropinisirung unverändert eintreten und sind in Folge dessen in der mehrfach erwähnten Abhandlung über das dentsche Aconitin der Behauptung A scharumow's entgegengetreten, der die genannten Wirkungen auf eine centrale Vagusreizung bezog. Beim Pseudaconitin begegnet man nun ganz entschieden einem Wirkungsstadium, das unmittelbar nach der Giftinjection auftretend nicht anders denn als der Ausdruck einer energischen centralen Vagusreizung gedeutet werden kann. Der Druck sinkt plötzlich, die Pulsfrequenz nimmt ab, kurz die Curven gleichen auf ein Haar denen, die man gewinnt, wenn man den N. vagus eines Thieres elektrisch reizt. Dieses Stadium fällt nun auch - folgerichtig - bei der Pseudaconitinvergiftung weg, wenn man vor der Vergiftung den NN. vagi durchschnitten hat oder es verschwindet, wenn man während seiner Dauer diese Operation vornimmt. Dadurch ist aber seine Beziehung zu einer centralen Erregung des Hemmungsvagus ausser Zweifel gesetzt. Niemals ist dieses Stadium bei der Pseudaconitinvergiftung von längerer Daner. 
Es macht vielmehr sehr bald einem Verhalten des Blutdrucks und der Pulsfrequenz Platz, das sich schwer beschreiben lässt und durch seine enorme Unregelmässigkeit charakterisirt ist. In der Regel steigen zunächst Druck und Frequenz beträchtlich und schwanken nun längere oder kîrzere Zeit in weiten Grenzen hin und her bis zuletzt unter stetigem und bedeutendem Sinken des Blutdrucks und Verschwinden der Pulscurve der Stillstand des Herzens in Diastole erfolgt. Die hierzu erforderliche Dose übersteigt auch für kräftige Hunde nicht 1 Milligramm, wenn man das Gift in die Jugularvene einspritzt. Künstliche Respiration und Curare müssen selbstverständlich angewandt werden, weil ohne dem die Respirationsstörungen die ganze Scene erfüllen wiirden. Die Prüfung des Verhaltens der Herznerven zeigt, dass fast unmittelbar nach dem Verschwinden des geschilderten ersten Stadiums der centralen Vagusreizung der Vagus seine Errregbarkeit verliert - die elektrische Reizung seines peripheren Stumpfes bleibt obne Wirkung auf Puls und Blutdruck. Auch hierin sahen wir eine Differenz von der Wirkung des deutschen Aconitins, dessen Einfluss auf den N. vagus der Säugethiere ein sehr wechselnder und unbestimmter ist.

Gegenüber dem vasomotorischen Centrum, seiner reffectorischen Erregbarkeit anf dem Wege sensibler Nerven sowohl als seiner directen Erregbarkeit verhalten sich Pseudaconitin und Aconitin identisch. Sensible Reize verlieren durch das Pseudaconitin sehr bald ihre Wirkung auf den Blutdruck curarisirter Thiere, der zuletzt in Folge einer directen Lähmung des Gefässnervencentrums fast bis zur Nulllinie sinkt.

Der diastolische Herzstillstand endlich ist dem durch das deutsche Aconitin erzeugten durchwegs analog.*)

Endlich bliebe mir noch die Aufgabe übrig, über einige Versuche zu referiren, die Ewers an Menschen iiber die locale Wirkung des Pseudaconitins aut die Haut angestellt hat. Er fand, dass der Stoff in alkoholischer Lösung auf die äussere Haut applicirt Tastund Temperaturempfindung bedeutend herabsetzt. Die Versuche wurden an intelligenten und sachkundigen Individuen mit dem Tastercirkel und dem ron Nothnagel für die Prüfung des Temperatursinns angegebenen Apparate angestellt.

Die angewandte Lösung des Giftes bestand aus gr.jj Pseudaconitin und $5 \mathrm{j}$ Alkohol. Die betreffenden Versuche theilen wir in extenso mit.

* Die genauen Zahlcnbelege für alle die im Vorstehenden aufgestellten Behauptungen sind in Ewers Schrift nachzusehen, die ich auf specielles Verlangen Fachgenossen gerne mitzutheilen bereit bin. 
Versuch 3ă. 7. April 9 h. Abends. A. P., stud. phil., 18 Jahre ait von gracilem Körperbau und zarter Haut. Der geringste Abstand der Zirkelarme, bei dem die Spitzen gesondert empfunden werden, schwankt an beiden Wangen zwischen 5 und $7 \mathrm{Mm}$. An beiden Wangen wird in der Temperaturscala von $35^{0}$ bis $39^{\circ} \mathrm{der}$ Unterschied von $0,5^{0} \mathrm{C}$. gefühlt. Die rechte Wange wird mit einer alkoholischen Aconitinlösung (gr.jj : $5 \mathrm{j}$ ) eingerieben.

8. April 9 h 30 Min. Morgens. A. P. giebt an, dass bald nach der Einreibung heftiges Brennen und Prickeln entstanden, welches auch augenblicklich noch, aber weniger intensiv bestehe. Dasselbe brennende Gefïhl habe er in einem verstärkten Maasse in dem rechten Auge empfunden, dessen Entstehnng er darauf zurückgeführt, dass er mit der Hand die eingeriebene Wange und gleich darauf das Auge berührt. Das obere Lid des reçhten Auges etwas geschwellt, die Gefässe der Conjunctiva injicirt. In der Weite der Pupillen keine Differenz. Versuch mit dem Tasterzirkel ergiebt als geringsten Abstand an der linken Wange $7 \mathrm{Mm}$., an der rechten $15 \mathrm{Mm}$. Der geringste empfundene Temperaturunterschied beträgt links $0,5^{\circ}$ C., rechts $1,4^{\circ} \mathrm{C}$. Ein und dasselbe Gefäss in rascher Aufeinanderfolge mit. der rechten und linken Wange in Berührung gẹbracht, wird, wie mit grosser Entschiedenheit angegeben wurde, auf der linken Wange bedentend wärmer empfunden. 9. April $8 \mathrm{~h}$ Abends. Das Prickeln und Brennen ist geschwunden and hat einem Gefühl von "Vertaubtheit" Platz gemacht. Injection der Conjunctivalgefässe nicht mehr vorhanden. Geringster Abstand der Zirkelspitzen rechts 13 , links $6 \mathrm{Mm}$. Geringster Temperaturunterschied, der auf der rechten Wange gemerkt wird, beträgt $1,1^{\circ} \mathrm{C}$.

Versuch 36. 10. April 10 h. Abends. C. H., stud. med. von kräftigem Körperbau, wird die rechte Wange mit der oben angegebenen Aconitinlösung eingerieben.

11. April 8 h. Abends. Das Prickeln und Brennen, sofort nach der Einreibung entstanden, danert noch fort. Temperaturunterschiede von 2,7 ${ }^{\circ} \mathrm{C}$., welche auf der linken Wange mit der grössten Präcision empfunden werden, werden an der rechten Wange nicht wahrgenommen. Geringster Abstand der Zirkelspitzen links 8, rechts $18 \mathrm{Mm}$.

13. April 9 h. Abends. C. H. giebt an, dass er an der rechten Wange nux noch ein ganz leises Prickeln' empfinde. Aufgefallen sei ihm die Erfahrung, dass bei Berührung der Wangen mit kalten metallischen Gegenständen letztere $\mathrm{ihm}$ an der rechten Wange weniger kalt erscheinen wie an der linken Wange. Geringster Abstand der Zirkelspitzen rechts 20, links $9 \mathrm{Mm}$. Geringster Temperaturunterschied, der gefühlt wird, rechts 2,80 C., links 0,90 C. Dasselbe Gefäss auf die rechte und linke Wange gebracht, wird stets anf der linken Wange wärmer empfunden.

15. April 2 h. Nachmittags. Prickeln und Brennen vollständig geschwunden. Geringster Abstand der Zirkelspitzen rechts 16, links $9 \mathrm{Mm}$. Temperaturunterschiede von 2,40 C. werden, links präcis empfunden, rechts nicht.

Versuch 37. 5. April 10 Uhr Vormittags. L. v. W., stud. med., 21 Jahre alt, von kräftigem Körperbau. Geringster Abstand der Zirkel- 
spitzen beträgt auf beiden Wangen $11 \mathrm{Mm}$. Temperaturunterschiede von $0,9 \circ \mathrm{C}$. werden an beiden Wangen empfunden. Die rechte Wange wird mit der bisher benuzten Lösung, die linke mit einer Lösung Merck'schen Aconitins von derselben Concentration eingerieben.

6. April 9 h. Morgens. Die subjectiven Empindnngen an der rechten Wange werden ebenso wie von den früheren Versuchsobjecten angegeben. An der linken Wange ist keine Wirkung verspürt worden. Geringster Abstand der Zirkelspitzen beträgt rechts 18 , links $10 \mathrm{Mm}$. Temperaturunterschiede von $2,30 \mathrm{C}$. werden rechts nicht empfunden, links sehr präeis.

Eine am Abend desselben Tages nochmals vorgenommene Prüfung der Tast- and Temperaturempfindung ergab dasselbe Resultat.

Aus diesen Versuchen geht mit Evidenz hervor, dass das Pseudaconitin, äusserlich applicirt, die Tast- und Temporaturempfindung herabsetzt, - eine Wirkung, die wohl auf einen lähmenden Einfluss dieses Giftes anf die, diese Sinneswahrnehmungen vermittelnden, nervösen Endapparate zurückzufithren ist. Diese Eigenschaft kommt nur dem aus Aconitum ferox bereiteten Alkaloid $\mathrm{zu}$, indem das deutsche Aconitin, das bekanntlich ans Aconitum napellus dargestellt wird, sich vollkommen unwirksam gegen die äussere Hant erwies.

Ueber den therapentischen Werth des Psendaconitins steht uns leider keine grössere Erfahrung zn Gebote. Das hier seltene Vorkommen von circumscripten, anf bestimmte Nervenbahnen beschränkten Neuralgien ist die Ursache, dass wir das Aconitin nur in eimem einzigen Falle haben therapentisch verwenden können. Dieser eine Fall betraf einen fünfzigjährigen Mann, der am 22. Februar in der Ambulanz der hiesigen Poliklinik erschien, über heftige Sehmerzen in der rechten Supraorbitalgegend klagend. Diese Schmerzen bestanden nach seiner Aussage mehrere Jahre trotz vielfach änsserlich und innerlich angewandter Medicamente. Eine anatomische Veränderung liess sich an der erkrankten Partie nicht nachweisen. Druck auf die der Incisura supraorbitalis entsprechende stelle wurde als höchst schmerzhaft empfunden. Diagnose: Neuralgia supraorbitalis. Die schmerzhafte Stelle wird mit der zu physiologischen Versuchen benutzten Pseudaconitinlösung eingerieben. Den 23. Februar gab Patient an, er habe während der Nacht ein so starkes Brennen empfunden, dass er gar nicht habe schlafen können. Von seinen Schmerzen habe er allerdings nichts gespürt. Den 26. Februar erschien er wieder auf der Klinik, um sich für die Arznei zu bedanken. Seine Schmerzen seien nicht wiedergekehrt. Mit dem Versprechen sich-sofort wieder vorzustellen, sobald jene auftreten sollten, verliess er die Ambulanz und hat sich bis zu diesem Augenblick nicht wieder gezeigt, so dass wir wohl miteiniger Wah rscheinlichkeit annehmen können, dass er auf die Daner von seinen Leiden befreit worden.

Wenngleich dieser eine unter dem Einfluss des Aconitins günstig verlanfene Krankheitsfall noch durchaus nicht die therapentische Wirksamkeit des Aconitins beweist, so dient er doch immerhin dazu, dieses Mittel zu weitern therapeutischen Versnchen zu empfehlen. 